REVISTA ANDALUZA DE ANTROPOLOGÍA.

NÚMERO 3: MIGRACIONES EN LA GLOBALIZACIÓN.

SEPTIEMBRE DE 2012

ISSN 2174-6796

[pp. 80-93]

http://dx.doi.org/10.12795/RAA.2012.i03.05

Fecha de recepción: 28/11/2011

Fecha de aceptación: 22/04/2012

\title{
ENTRE LAS DICTADURAS Y EL PETRÓLEO: LAS MIGRACIONES TRANSNACIONALES DE GUINEA ECUATORIAL
}

YOLANDA AIXELÀ

Institució Milà i Fontanals del CSIC

\section{Resumen}

El objetivo de este artículo es el de presentar una genealogía de las migraciones guineoecuatorianas desde mediados del siglo XX hasta la actualidad. La emigración guineana se inició a finales del período colonial y estuvo motivada por temas políticos y formativos, causas que continuaron vertebrándola tras la instauración de las dictaduras de Macías y de Obiang. Ambas se combinaron con las minoritarias migraciones laborales. El hallazgo de petróleo a mediados de la década de los 1990 actuó como "efecto reclamo" y marcó el inicio de flujos migratorios inversos, apreciables a partir del año 2000, en unas inmigraciones que en parte protagonizarían algunos de los guineanos que se habían asentado en España o algunos de sus descendientes. La integración de la población de Guinea era difícil, tanto en los nuevos contextos de asentamiento en el extranjero como cuando regresaron a su país, pero los que han regresado a Guinea Ecuatorial por la crisis económica mundial, principalmente de países en los que han disfrutado de la ciudadanía democrática, tienen que enfrentar vivir en un país que no respeta los "derechos" por el sistema político dictatorial. 
Palabras clave: Guinea Ecuatorial, migraciones transnacionales, exilio, estudios, dictadura, petróleo.

\begin{abstract}
The aim of this paper is to present a genealogy of Equatorial Guinean migration from mid- twentieth century to the present. Equatorial Guinean's emigration began in the late colonial period and was motivated by political issues and training, reasons that continued after the establishment of dictatorships of Macias and Obiang. Both were combined with the labor Migration. The discovery of oil in mid-1990 acted as a "claim effect" and signaled the start of reverse migration, observed from the year 2000, in part started by some of the Guineans who had settled in Spain or some of their descendants. The integration of Guinean people was difficult both in the new contexts of settlement abroad as when they returned to their country, but those who have returned to Equatorial Guinea by the global economic crisis, largely from countries where they have enjoyed democratic citizenship, live in a country that no respect to "rights" and "duties" by dictatorial political system.
\end{abstract}

Keywords: Equatorial Guinea, transnational migration, exile, studies, dictatorship, oil.

\title{
1. EL INTERÉS POR LAS MIGRACIONES GUINEOECUATORIANAS
}

La historia de las migraciones transnacionales de guineanos desde mediados del siglo XX y hasta la actualidad ha sido escasamente estudiada de forma monográfica salvo por Fons (2002, 2004), Ugarte (2009), Sipi (2000a, 200b, 2004, 2010), Vi-Kome (2000) y Ndongo $(1977)^{1}$. Los textos son sorprendentemente insuficientes si se tiene en cuenta que Guinea Ecuatorial fue excolonia española hasta 1968, y que España ha reunido la comunidad guineoecuatoriana más numerosa fuera de Guinea, con un flujo migratorio especialmente notable con la instauración de la violenta dictadura de Macías Nguema cuando en España aún regía la dictadura de Francisco Franco. Es probable que esta inexplicable invisibilidad se haya debido, no sólo al "desinterés" de la política española por esta comunidad y por los acontecimientos que abatían su país, sino también por el bajo número de guineoecuatorianos en España en relación a otros grupos migrantes, así como a su rápida adopción de la nacionalidad española. Creemos que los extendidos criterios cuantitativos deberían en este caso substituirse por los justificados criterios cualitativos. Por otro lado, las complicaciones para obtener un visado para viajar a Guinea o la imposibilidad de obtener estadísticas del país, una de las herramientas de apoyo más empleadas por los científicos sociales, ha acabado por hacer desistir a muchos

1. Este artículo se inserta en el marco del Proyecto I+D "La gestión de la diversidad cultural y el impacto sociopolítico de las migraciones transnacionales en dos ex-colonias españolas: Guinea Ecuatorial y Marruecos" (HAR2011-22752) dirigido por Y. Aixelà de la Institució Milà i Fontanals del Consejo Superior de Investigaciones Científicas. 
investigadores de estudiar un colectivo hermético y celoso de su intimidad. Las cuestiones señaladas concretan los objetivos de este texto: establecer causas y periodificaciones de las migraciones guineanas desde una perspectiva transnacional, con datos presentados de forma relacional ${ }^{2}$, obtenidos durante un trabajo de campo multisituado, basado en entrevistas y observación participante, iniciado en 2004 y desarrollado de manera intensiva desde 2008 y hasta la actualidad, en Guinea Ecuatorial y España, combinando el trabajo de campo con estancias breves en Sudáfrica, Holanda, Suiza, Gran Bretaña y Marruecos en 2009.

Cabe señalar que las migraciones guineanas desde mediados del siglo XX y hasta la actualidad están estrechamente vinculadas a factores de diferente índole, como son los políticos, los formativos y los laborales ${ }^{3}$.

Los primeros flujos migratorios de guineanos fuera de Guinea Ecuatorial (al margen de los captados por las porosas fronteras de Camerún y Gabón) se debieron a una emigración de carácter marcadamente político muy vinculada a la historia de Guinea Ecuatorial. Así, se desarrollaron en el marco de ciertos acontecimientos históricos tales como son, por un lado, la reclamación del fin de la colonización española lo que llevó a la huída a la minoría guineana que la defendía y, por otro, la instauración de la dictadura de Francisco Macías Nguema (1969) tras la llegada a la Independencia colonial (1968) que promovió el exilio de miles de personas y que también se reprodujo, aunque en menor número, durante el establecimiento de la dictadura de Teodoro Obiang Nguema (1979).

Estas emigraciones por motivos políticos se simultanearían con unas escasas y selectivas emigraciones de estudios en la década de 1950, mayormente a España en el caso de las primeras emergidas en los lustros anteriores al fin de la colonización, pero más numerosas y con otros destinos migratorios formativos a Europa, Asia y América bien dispares, tras la instauración de la Independencia colonial, dado que eran promocionadas por los regímenes dictatoriales guineanos (especialmente el de Obiang) con el objetivo de garantizar la formación de ciertos cuadros del gobierno para el control de los recursos y también de dotar de formación cualificada universitaria a algunos guineanos que debían regresar a un país carente de población con formación académica completa. En la década del 2000, un lustro más tarde del hallazgo del petróleo de mediados de

2. Emma Martín Díaz (2008) ha insistido en diferentes publicaciones sobre la necesidad de profundizar y reorientar algunas perspectivas sobre las migraciones de cara a valorar factores que con la perspectiva transnacional cobran relevancia: "Esto implica revisar conceptos claves como sociedad y cultura, en la medida en que han cambiado los soportes básicos para su análisis: básicamente el territorio y la forma político-administrativa que caracterizó la entrada de las sociedades en la "primera modernidad" a escala global, el Estado-nación".

3. El contenido de este artículo forma parte de una investigación más amplia que se puede consultar en Aixelà (2011) Guinea Ecuatorial: ciudadanías y migraciones transnacionales en un contexto dictatorial africano. Vic: Ceiba. Una primera aproximación se ofreció en Aixelà (2010a). 
la década de 1990, se crearon flujos inmigratorios hacia Guinea Ecuatorial, no sólo de personas de distintas nacionalidades procedentes de América, Europa, Asia y África, sino también de guineanos o descendientes de éstos, establecidos en España u otros países; todos buscaban la prosperidad en un país con importantes recursos petrolíferos (Denantes, 2009; Campos, 2011) y si bien los primeros en llegar tenían una alta cualificación, la crisis económica en España y en otros países donde se asentaron, sigue promoviendo un regreso incómodo, por cuanto han vivido mayoritariamente en democracias y se establecen en un sistema político dictatorial.

Señalar que estos tres flujos migratorios -la emigración previa a la descolonización, la emigración que huía de las dictaduras y la inmigración que regresó tras el hallazgo del petróleo- coexistieron con flujos migratorios inversos y diversos, aunque no serían los más representativos de cada período. Así, se pueden destacar los guineanos que migraron por motivos laborales hacia Camerún y Gabón o los guineanos que establecieron su matrimonio mixto en España.

A continuación, se van a presentar las distintas migraciones guineoecuatorianas con el objetivo de establecer una clasificación que amplíe y clarifique la información existente sobre éstas.

\section{LA EMIGRACIÓN DEL FIN DEL PERÍODO COLONIAL: DE 1950 A 1968}

La primera emigración de exiliados políticos fue minoritaria. Se trataba de guineanos que, siguiendo el efecto cadena del inicio de la descolonización europea en la década de 1950 en África, empezaron a reclamar la salida de los colonizadores españoles, constituyendo asociaciones opositoras más o menos estructuradas en Guinea Ecuatorial. Una parte de los que tuvieron que huir, se dirigieron a países africanos vecinos, creando plataformas que, aunque precarias, demandaban el fin de la colonización de su país. Esta movilización se constituyó especialmente en los países africanos en los que se asentaron, tales como fueron Camerún, Gabón y Nigeria. Una pequeña parte de estos opositores continuaría viaje hacia países europeos, buscando mayor eco a sus reclamaciones sociopolíticas, lo que unos pocos consiguieron en Suiza, lugar de referencia de los derechos humanos, y no tanto en España donde la dictadura de Franco no quería manifestarse contra la dictadura de Macías, prohibiendo incluso que se informara en España sobre el país en el último tramo franquista, catalogando todo lo relacionado con Guinea Ecuatorial "Materia reservada". Mientras este pequeño sector de guineanos en Guinea reclamaba el fin de la colonización, otros guineanos consiguieron desplazarse a España para realizar estudios, gracias a las presiones ejercidas al régimen colonial y al estamento misional. La migración de estudios era necesaria, primero, porque la escolarización en Guinea no llegaba a toda la población y puso límites a la formación de los guineanos durante la mayoría del período colonial y, segundo, porque la formación era incompleta, precaria y seguían sin existir universidades 
(la UNED inició su formación en 1981 y la Universidad Nacional de Guinea Ecuatorial se fundó en 1995). Ante esta realidad, la única solución para todos aquellos que deseaban obtener una formación más completa era la emigración, incluida la elite negroafricana fernandina de Bioko ${ }^{4}$ que, por lo general, ya mandaba a sus hijos a estudiar al extranjero incluido Gran Bretaña. Los canales que se emplearon para emprender la migración de estudios de los guineanos fueron, al principio, los eclesiásticos ya que se hacían cargo del estudiante en España, tanto de su formación como de su alojamiento y manutención. Eso sí, la selección de candidatos estaba supeditada a los criterios de los misioneros en Guinea que, a su vez, podían ser aconsejados desde la propia colonia española residente en el país. Años más tarde, se sumarían algunas becas de estudios que el gobierno español puso al servicio de algunos guineanos y nuevamente su selección recaería en los consejos de los gestores coloniales. Por supuesto, una parte de los que habían utilizado el sistema de becas para estudiar en el extranjero no regresarían, bien porque tras su finalización se asentaron, bien porque su interés era establecerse en España.

\section{LA EMIGRACIÓN CON LA INSTAURACIÓN DE LAS DICTADURAS GUINEANAS: DE 1968 A LA ACTUALIDAD}

La llegada a la Independencia colonial en octubre de 1968 y el establecimiento de la primera dictadura de Macías Nguema en 1969, caracterizada por la violencia indiscriminada y la impunidad del régimen, llevó a miles de guineanos al exilio, no sólo africano, sino también español. Algunos especialistas estimaron que el porcentaje de guineanos y guineanas que huyeron de Guinea Ecuatorial no fue menor de la cuarta parte de la población, sabiendo que Guinea tenía 245.989 habitantes en el Censo de $1960^{5}$, y 375.000 personas en 1981 (Cabana, 1995:9) ${ }^{6}$. En total, marcharían cerca de 100.000 personas desde la década de 1970 y hasta finales de la década de 1990. La gran mayoría de los emigrantes se fueron sin ningún tipo de red familiar de apoyo en tanto que sería precisamente su generación la que crearía esas redes de acogimiento que facilitarían la emigración de sus parientes cercanos y de las subsiguientes generaciones.

Los que se establecieron en España se integraron en una sociedad en la que aún pesaba la dictadura de Francisco Franco y donde la experiencia de recibir migraciones externas era escasa. Su inserción en el nuevo contexto careció pues de apoyo y sensibilidad gubernamental al tiempo que en España se silenciaban las atrocidades que cometía el régimen de Macías Nguema en Guinea. Debe incidirse en que la emigración del exilio

4. Los fernandinos son descendientes de esclavos libertos, concretamente de los esclavos liberados en la isla de Bioko por los británicos. Constituyeron una verdadera elite poderosa en el país.

5. Anuario Estadístico de España de 1969, p.460.

6. En 2007 la población se estimó en 551.201 (Seibert, 2008) Debe señalarse que ningún gobierno guineoecuatoriano ha realizado un censo aceptable de población en el período postcolonial y que las estadísticas oficiales más fiables se remontan a períodos previos de la Independencia colonial. Sobre el particular se puede consultar entre otros Anglada (2011). 
fue más numerosa durante la dictadura de Macías que durante la de Obiang, aunque no se detuvo. Las personas y familias que se exiliaron al extranjero esperaban encontrar un futuro mejor y sólo una pequeña parte pensaba que podría regresar en poco tiempo para reprender su vida en el país. Una situación atípica fue la que vivió el pequeño grupo de guineanos que se encontraban en España cursando sus estudios cuando llegó la independencia y estalló la dictadura, ya que se encontraron en unas circunstancias precarias y delicadas porque mientras Macías les instaba a regresar so pena de perder la nacionalidad guineana, sabían que volver a un país en el que había estallado una violencia indiscriminada de Estado no sería seguro para ellos. Así que la mayoría de los estudiantes se quedó perdiendo todo el contacto con sus familias, sin saber si estaban vivos o muertos, y asumiendo que deberían sobrevivir en una España que no les protegió ante la pérdida de becas y precariedad económica. De hecho, algunos tuvieron estatuto de apátridas durante un breve período por la falta de compromiso del gobierno español para prestarles ayuda. El testimonio de Davis (2009) nos aproxima la desazón colectiva.

Mientras se producía esta marcha de miles de personas al exilio, la dictadura guineana reestructuró los canales de emigración de estudios existentes antes de la Independencia, y abrió nuevos destinos formativos, básicamente con aquellos gobiernos que habían establecido complicidades con el sistema político de Macías. Así, la ex Unión Soviética, Cuba, China y Corea del Norte, serían los destinos preferentes a los que el régimen enviaría a los privilegiados seleccionados para cursar estudios. Con el hallazgo del petróleo de mediados de la década de 1990, cambió el marco de relaciones internacionales de Guinea, ampliando su nómina de estrechas relaciones no sólo con socios preferentes como USA y Francia, sino también con otros países que deseaban garantizar su potencialidad económica en Guinea, como por ejemplo la propia China. Este cambio generó que los estudiantes guineanos pudieran optar a becas en numerosos países de América, Asia, África y Europa (Aixelà 2011).

Cabe añadir que durante este período también hubo guineanos que emigraron por trabajo si bien esta motivación no fue en absoluto principal. De hecho, la mejora laboral ya formaba parte de las esperanzas de todos los que se marcharon dado que salían de un país donde la colonización sólo había fomentado las explotaciones agrícolas sin favorecer la aparición de otras profesiones más cualificadas y, por supuesto, la redistribución de los recursos entre la población (Kabunda, 2007; Creus, 2007). Una gran parte de los que tuvieron motivaciones laborales se establecieron en Camerún y Gabón.

\section{LAS INMIGRACIONES A BIOKO TRAS EL HALLAZGO DEL PETRÓLEO: DE 1998 A LA ACTUALIDAD}

El hallazgo de petróleo a mediados de la década de 1990 significó un profundo cambio en la economía guineana. Si durante la colonización la isla de Bioko había demandado fuerza 
de trabajo no especializada para las plantaciones coloniales de cacao y café procedente, sobre todo, de África central y occidental -flujos migratorios que con la Independencia se detuvieron-, cuando se halló petróleo en Guinea Ecuatorial y el país se convirtió en uno de los principales productores de crudo del continente africano, se inició una nueva demanda de mano de obra, cualificada y con conocimientos altamente tecnificados. Y aunque parte de la misma la proporcionaron las propias empresas extractoras de Estados Unidos y Francia, mucha otra provino de América y Europa.

La necesidad de contar con mano de obra especializada para construir plataformas petrolíferas, mantenerlas y gestionarlas, así como para atender todos los negocios que rodeaban la extracción del cruzo de mediados de la década de 1990 en adelante, empezó a ejercer de notable "efecto llamada" de inmigrantes de países diversos ${ }^{7}(7)$, sobre todo a partir de los primeros años de la década del 2000, por lo que el interés por la riqueza de Guinea se extendió también a agricultores y pescadores africanos que buscando un deseo de mejora que mayormente no se cumpliría, llegaban a Guinea incluso, a pie, cruzando las fronteras de Camerún y Gabón, o en patera, a la costa de la isla de Bioko. Más importante es, si cabe, que a partir de 2001 empezaron a regresar también los guineanos que se hallaban asentados en países europeos y en Estados Unidos, especialmente aquellos residentes en España. Los guineanos procedentes de España constituyen un grupo formado especialmente por los descendientes de los que se marcharon en los años 1970, personas que, como sus ascendientes, tienen mayoritariamente doble nacionalidad, española y guineana (ya que España no ha exigido la renuncia de la guineana cuando les ha otorgado su nacionalidad), lo que no han exhibido porque, para prosperar en la Guinea Ecuatorial de hoy, da mayor garantía hacerlo como guineano que como español. Añadir que esta generación muestra las contradicciones entre la dictadura y la democracia porque aquellos que regresan han crecido o se han desarrollado en un sistema político de libertades de las que carecen a su regreso. Éstos también utilizan las redes familiares para una integración social más rápida y están primando la redistribución selectiva y no la generalizada.

Esta nueva realidad económica, que no política, ha llevado a que actualmente en la isla de Bioko (que es donde se reúnen las explotaciones petrolíferas), y especialmente en la ciudad de Malabo, convivan personas de numerosos orígenes geográficos y culturales, por lo que junto con los guineanos bubis, fang, ndowe, bissio y annoboneses, hay europeos de España, Francia, Rusia; asiáticos de China, Corea, Líbano, Siria, Vietnam;

7. El hallazgo de petróleo a mediados de la década de 1990 supuso un cambio substancial de Guinea Ecuatorial a nivel del Producto Interior Bruto. Esta mejora que conllevó el potencial energético (el cuarto extractor de todo el continente africano) supuso un empuje en el marco de las Relaciones Internacionales dado que numerosos países, consolidados y emergentes, deseaban establecer relaciones económicas con Guinea. Sin embargo estos cambios no se han reflejado en el total de la ciudadanía que sigue viviendo en la pobreza. 
y africanos de Egipto, Marruecos, Gabón, Camerún, Nigeria, Ghana, Sudáfrica, etc. En la capital de Guinea Ecuatorial se podrían aplicar los marcos teóricos desarrollados en Europa y países emergentes sobre multiculturalismo y diversidad cultural (Aixelà 2010b).

\section{LA COMUNIDAD GUINEANA EN EL EXTRANJERO Y LA EMERGENCIA DE UNA SOCIEDAD CIVIL TRANSNACIONAL}

Las especiales circunstancias que motivaron la salida de miles de personas de Guinea Ecuatorial en dirección a países africanos limítrofes, a España, y en menor medida a otros países europeos y Estados Unidos, creó un asentamiento guineoecuatoriano disperso y poco cohesionado. Por ejemplo, las redes migratorias de guineanos no estuvieron disponibles hasta la década de 1980, tras la muerte de Macías, cuando los primeros emigrantes se habían asentado en los diferentes contextos tras su marcha a principios de la década de 1970. Aunque Obiang continuó presidiendo un régimen dictatorial represor, los canales de comunicación del país se abrieron paulatinamente permitiendo que los guineanos residentes en España consiguieran contactar con sus familias, haciéndoles saber su localización y facilitando una posible emigración de parientes consanguíneos o afines, antes complicada. La dispersión guineana que favoreció la desunión de la comunidad guineoecuatoriana, se vio reforzada por un clima generalizado de desconfianza gestado en tiempos de Macías y que había hecho mella en una población temerosa de ser denunciada por sus compatriotas, preocupada por las represalias que las noticias de sus actitudes y actividades en situación migratoria pudieran ocasionar sobre sus familiares en Guinea. Esta tensa angustia se ha venido manifestando hasta la actualidad.

A estos factores que han impedido la cohesión de la comunidad guineana transnacional se ha sumado otro factor, la influencia del régimen dictatorial más allá de sus fronteras, especialmente a partir de la década de los 1990 y tras el hallazgo del petróleo, dado que existen canales de recogida de información sobre los guineanos en el extranjero, tanto institucionales -a través de las respectivas embajadas y consulados-, como asociativos cercanos al partido del gobierno (Partido Democrático de Guinea Ecuatorial), como individuales (personas afectas al régimen). Debe destacarse que la mayoría de las personas vinculadas al Gobierno realizan constantes viajes a España; muchos tiene tienen a sus hijos cursando estudios o a sus familiares residiendo con un alto nivel de vida en diferentes capitales de provincia como Madrid, Barcelona, Alicante o Valencia, donde se han comprado inmuebles. Todos los factores mencionados permiten pues apuntar que la elite dictatorial y el propio régimen también son transnacionales ${ }^{8}$. Como señalaban acertadamente Kech y Sikkink (1998:199) "scholars theorizing about transnational relations must grapple with the multiple interactions of domestic and international politics as sources of change in the international system. The blurring of boundaries between

8. Aixelà (2011:90). 
international and domestic arenas has long been evident in international and comparative political economy, but its relevance for other forms of politics is less theorized"9. En esa línea, Slaughter (1995:509) había señalado que "liberal international relations theories applies to all the States. Totalitarian governments, authoritarian dictatorships, and theocracies can be all depicted as representatives of some subset of actors in domestic and transnational society, even if it is a very small slice".

Los aspectos mencionados son clave para explicar la falta de cohesión de la comunidad guineana en el extranjero, especialmente en España, y la lenta emergencia de una sociedad civil guineana transnacional. Aspectos como la desconfianza política, las diferencias étnicas, la afinidad o no con el gobierno de Guinea, la participación o el desprecio por el régimen, entre otros, no han facilitado la posible creación de estructuras, formales o informales, que canalizaran un generalizado descontento. Y es que la sociedad civil guineana no ha tenido oportunidad de emerger en Guinea por la represión y el control del régimen, pero sí está germinando en situación migratoria. Ahora bien, es destacable su debilidad y precariedad por cuanto carece del apoyo de los jóvenes nacidos en el extranjero y también de los formados fuera de su país que han retornado. Los primeros porque sus familiares les han salvaguardado del terror y la dictadura, habiéndose integrado, con más o menos fortuna, en los países en los que viven. Los segundos porque mayoritariamente (aunque no siempre) están afectos al régimen y no (se) plantean un cambio en el sistema político, sino regresar con una buena formación para poder beneficiarse de la riqueza generada por el petróleo en el país.

Esta situación ha llevado a que la sociedad civil guineana también esté deslocalizada fuera de las fronteras de Guinea Ecuatorial, salvo un reducido número de personas residentes en Guinea carentes de conexión y estructura organizativa, y sea transnacional. En ese sentido se había manifestado Slaughter (1995:509) para quien el tipo de régimen es importante porque gobiernos autoritarios "can stunt the growth of domestic and transnational civil society".

\section{GUINEOECUATORIANOS EN ESPAÑA}

La comunidad guineana más numerosa fuera de Guinea Ecuatorial se asentó en España. El período de llegada coincidió con las migraciones de exilio y de estudios descritas en anteriores apartados, por lo que se tienen noticias de los guineanos en el país desde la década de 1950 (Mbare Ngom, 1996), aunque su presencia no sería numéricamente importante hasta primeros de la década de los 1970, coincidiendo con el punto álgido de la indiscriminada violencia de la dictadura de Macías. Es por ello que, como mencionamos,

9. Al respecto, Kech y Sikkink (1998: 200) explicitaban que: "We suggest that scholars of international relations should pay attention to network forms of organization - characterized by voluntary, reciprocal and horizontal exchanges of information and services... What is distinctive about the networks we describe here is their transnational nature, and the way they are organized around shared values and discourses". 
el establecimiento de las redes migratorias familiares no se consolidó hasta la década de los 1980 asegurando así, justo con la instauración de la dictadura de Obiang, una cierta capacidad de ayuda y solidaridad entre parientes para favorecer la emigración a la exmetrópolis.

Son numerosas las dificultades para proporcionar cifras concretas de los guineoecuatorianos en España y también de su profusa llegada tras la Independencia de Guinea, tal como constaté en Aixelà (2011), y no tanto porque los territorios se considerasen provincias españolas ya que el estatus no reconoció a sus pobladores plenos derechos como españoles. Entre las diversas causas destaca que "es a partir de esa fecha, 1970, cuando hubo un alud de guineoecuatorianos emigrando hacia España, los cuales con una regularización ágil y una rápida concesión de la nacionalidad española, pasarían a engrosar la ciudadanía española sin poder distinguirlos del total. Resulta casi imposible localizarlos en las estadísticas de población como guineoecuatorianos ya que las cifras siguen siendo muy bajas respecto a las expectativas de población que salió de Guinea Ecuatorial precipitadamente, parte de la cual se dirigió a España" (Aixelà, 2011:83).

De hecho, la adquisición de la nacionalidad también fue un factor clave para evaluar el grado de integración en la sociedad española y, especialmente, su posicionamiento respecto a Guinea Ecuatorial, ya que los guineanos demandaron mayoritariamente la nacionalidad española, trasla residencia o el amparo de asilo. Así, el Colectivo Ioé (1999:56) recogía algunos datos sobre 1996 señalando que un 51\% de los guineoecuatorianos extranjeros residentes en España habían solicitado la nacionalidad, y también señalaba el Colectivo Ioé (1999:72) que "durante estos últimos años los solicitantes proceden principalmente de Rumania, Cuba, Irán, Liberia, Argelia y Guinea Ecuatorial. En cambio, quienes más habitualmente obtienen la condición de asilados son cubanos, guineanos ecuatoriales y argelinos". Un factor integrador de los recién llegados en España, tras la instauración de la primera dictadura en 1969, fue recogido por Domínguez (1992:307n) quien consideraba que pudieron ser las Leyes de 30 de diciembre de 1969 y de 15 de enero de 1970, que establecían su equiparación respecto a los trabajadores nacionales.

En general, los guineoecuatorianos en España, como en otros estados europeos, han constituido un colectivo que ha adquirido un alto nivel de estudios, aunque no todos han conseguido alcanzar profesiones liberales y una posición acomodada ya que han tenido que enfrentarse a un latente racismo y xenofobia que no siempre permitió que la totalidad se integrase en plenitud. Uno de los efectos positivos de esa relativa integración guineana en los países receptores podría beneficiar a Guinea Ecuatorial ya que la conciencia de ser/sentirse guineoecuatoriano ha seguido presente en una parte de estos migrantes transnacionales, que aunque no muy amplia, está permitiendo la aparición de una emergente sociedad civil guineoecuatoriana que reside, eso sí, fuera de las fronteras de Guinea Ecuatorial. Con un nivel sociocultural elevado y también una clara conciencia 
de quiénes son, buena parte de este colectivo maneja argumentos sólidos sobre el porqué no están viviendo, aún hoy, en Guinea Ecuatorial. Por ello, una de las cuestiones más importantes que afloraron en la investigación es que la sociedad civil guineana se asienta fuera de las fronteras guineanas y es transnacional, disponiendo de una cierta influencia en su contexto de origen.

\section{HACIA UNA VISIBILIZACIÓN DE LAS MIGRACIONES GUINEOECUATORIANAS}

La falta de información sobre el colectivo guineoecuatoriano residente en España y sobre los españoles de origen guineoecuatoriano, ha acabado justificando un aparente desinterés en profundizar sobre la presencia española en Guinea Ecuatorial durante la colonización y tras la Independencia, y en conocer las particularidades guineanas, hechos que han derivado en un desconocimiento sobre sus problemáticas en España, en parte sujetas al racismo y a la xenofobia (Aixelà, 2011), y también en la ignorancia sobre las reclamaciones sociopolíticas de disidentes y opositores, sin poder favorecer otro tipo de relaciones de Estado entre España y el régimen guineoecuatoriano más allá de los intereses económicos. Estos factores han venido debilitando la cohesión de la comunidad guineana transnacional asentada en el territorio español, comunidad carente de visibilidad y de apoyo institucional generalizado. Sin embargo, conocer la historia compartida entre Guinea Ecuatorial y España, debe pasar forzosamente por analizar las migraciones guineanas y por comprender las razones que motivan una migración que continua a caballo entre la huída de los sistemas dictatoriales y el beneficio indirecto del petróleo. 


\section{REFERENCIAS BIBLIOGRÁFICAS}

Aixelà, Yolanda (2011) Guinea Ecuatorial: Ciudadanías y migraciones transnacionales en un contexto dictatorial africano. Vic: Ceiba Edicions.

Aixelà, Yolanda (2010a) “The impact of transnational migrant networks in Equatorial Guinea from the point of view of gender", Exploratory Workshop of the European Science Foundation “Transnational Lives”. Disponible en: http://www.tlnetwork.ics.ul.pt/ textos/The $\% 20 \mathrm{impact} \% 20$ of $\% 20$ the $\% 20$ transnational $\% 20$ network $\% 20 \mathrm{in} \% 20$ the $\% 20$ Equatorial\%20Guinea\%20case\%20in\%20a\%20gender\%20view Yoland\%20Aixelà.pdf

Aixelà, Yolanda (2010b) "La reflexión postcolonial en contextos urbanos de alta diversidad cultural: Su aplicación al caso africano de Malabo (Guinea Ecuatorial)”. 7º Congresso Ibérico de Estudos Africano (Lisboa, 9-11 de septiembre de 2010). Disponible en: http://repositorioiul.iscte.pt/bitstream/10071/2310/1/CIEA7 17 AIXEL\%C3\%80 La\%20reflexi\%C3\%B3n\%20postcolonial\%20en\%20contextos\%20urbanos.pdf

Anglada, Josep (2011) “Un país sin estadística”, junio 2, Fundación Sur. Disponible en: http://www.africafundacion.org/spip.php?article9279 [Consultado julio 2011].

Campos, Alicia (2011) Petróleo y estado postcolonial: transformaciones de la economía política en Guinea Ecuatorial, 1995-2010. Implicaciones para la coherencia de las políticas españolas. Fundación Carolina - Avances de Investigación n 45. Disponible en: http:// www.fundacioncarolina.es/esES/publicaciones/avancesinvestigacion/Documents/AI5 $\underline{4}$. pdf [Consultado agosto 2011]

Colectivo IOÉ (1999) Inmigrantes, trabajadores, ciudadanos. València: Universitat de València.

Creus, Jacint (2007) "Guinea Ecuatorial. Vigilante y amorosa: colonización, poscolonización y ejercicio de poder". En Iniesta (ed.) La frontera ambigua. tradición y democracia en África. Barcelona: Bellaterra, pp.225-244.

Davies Eiso, Juan Manuel (2009) "La generación del 68. Sueño perdido, realidad recuperable". En Sampedro y Moliner (eds.) International Conference: Between three continents: Rethinking Equatorial Guinea on the Fortieth Anniversary of its Independence of Spain. New York: Hofstra University, 13p. Disponible en: http://www.hofstra.edu/pdf/ Community/culctr/culctr guinea040209 IXAeiso.pdf [Consultado enero 2010].

Denantes Teulade, Samuel (2009) Malabo, le nouvel Eldorado pétrolier de l'Afrique. Paris: L'Harmattan. 
Domínguez, Josefina (1992) “Canarias en el panorama internacional de la movilidad poblacional". Vigueta 0, pp.293-308.

Fons, Virgínia (2004) Entre dos aguas. Etnomedicina, proceación y salud entre los ndowé de Guinea Ecuatorial. Vic: CEIBA.

Fons, Virginia (2002) "Historia de un viaje sin retorno. Proceso migratorio de la población de Guinea Ecuatorial a España”. En L. Ondo, A. Bokesa, M. Liniger-Goumaz (dir.) Misceláneas Guineo Ecuatorianas I. Del Estado colonial al Estado dictatorial. Editorial Tiempos Próximos \& Les Éditions du Temps, pp. 109-120.

Kabunda, Mbuyi (2007) “África. Respuestas a los desafíos de la globalización”. En Iniesta (ed.) La frontera ambigua. Tradición y democracia en África. Barcelona: Bellaterra, pp.3561.

Kech, Margaret; Sikkink, Kathryn (1998) Activists beyond borders. New York: Cornell University Press.

Marcus, George E. (1995) "Ethnography In/Of the World System: the Emergence of Multi- Sited Ethnography”. Annual Review of Anthropology, vol. 24, pp. 95-117.

Martín Díaz, Emma (2008) “El impacto del género en las migraciones de la globalización: mujeres, trabajos y relaciones interculturales”. Scripta Nova XII, 270 (133). Disponible en: http://www.ub.edu/geocrit/sn/sn-270/sn-270-133.htm [Consultado junio 2010]

Mbare Ngom, Faye, (1996) Diálogos con Guinea. Panorama de la literatura guineoecuatoriana de expresión castellana a través de sus protagonistas. Madrid: Labrys.

Ndongo, Donato, (1977) Historia y tragedia de Guinea Ecuatorial. Madrid: Editorial Cambio 16.

Sipi, Remei (2010) Genealogies femenines: les dones immigrades a Catalunya. 20 anys d'associacionisme en femení. Barcelona: Yemanjà.

Sipi, Remei (2004) Inmigración y género. El caso de Guinea Ecuatorial. Donostia: Gakoa. Sipi, Remei (2000a) "Las mujeres subsaharianas en el territorio español”. Mugak 9/10, pp. 23-26.

Sipi, Remei (2000b) “Las asociaciones de mujeres ¿agentes de integración social?", Papers 60, pp. 355-364

Slaughter, Anne-Marie (1995) “International Law in a world of liberal states". European Journal of International Law 6, pp.503-538. 
Ugarte, Michael (2009) Africans in Europe: The Culture of Exile and Emigration from Equatorial Guinea to Spain. Illinois: University of Illinois Press.

Vi-Kome, Inongo (2000) La emigración negroafricana: tragedia y esperanza. Culturas alternativas. Barcelona: Ed. Carena. 